Research Paper

\title{
Microbubbles Enhance the Antitumor Effects of Sino- porphyrin Sodium Mediated Sonodynamic Therapy both In Vitro and In Vivo
}

\author{
Haiping Wang, Pan Wang, Li Li, Kun Zhang, Xiaobing Wang ${ }^{\bowtie}$, Quanhong Liu ${ }^{\circledR}$ \\ Key Laboratory of Medicinal Resources and Natural Pharmaceutical Chemistry, Ministry of Education, National Engineering Laboratory for Resource \\ Developing of Endangered Chinese Crude Drugs in Northwest of China, College of Life Sciences, Shaanxi Normal University, Xi' an 710062, Shaanxi, \\ China. \\ $\triangle$ Corresponding authors: Xiaobing Wang and Quanhong Liu. E-mail: Xiaobing Wang, wangxiaobing@snnu.edu.cn; Quanhong liu, \\ 1shaof@snnu.edu.cn. Telephone: +86-29-85310275
}

(c) 2015 Ivyspring International Publisher. Reproduction is permitted for personal, noncommercial use, provided that the article is in whole, unmodified, and properly cited. See http://ivyspring.com/terms for terms and conditions.

Received: 2015.05.27; Accepted: 2015.07.20; Published: 2015.11.15

\begin{abstract}
Objectives: To evaluate the anti-cancer effect of sonodynamic therapy combined with microbubbles both in vitro and in vivo.

Methods: Cell viability was measured by 3-(4, 5-dimethylthiazol-2-yl)-2, 5-diphenyl tetrazolium bromide and guava viacount assays. Annexin V-FITC/PI staining was adopted to analyze cell apoptosis rate. FD500 uptake assay was performed to assess cell membrane permeability changes. Tumor weight, mice weight and the visual image of tumor size were used to reflect the anti-tumor effect of this combined method. Histological change of tumor tissue after different treatments was measured through hematoxylin and eosin (H\&E) staining.

Results: Microbubbles can significantly enhance the cytotoxicity and necrocytosis rate induced by SDT treatment. Increased cell membrane permeability and more uptake of DVDMS were founded in SDT combined with microbubbles group. For in vivo experiments, SDT with microbubbles can significantly reduce tumor weight and size with pimping difference of mice weight compare with other treatment groups. In addition, microbubbles notably improved tumor tissue destruction caused by ultrasound and SDT treatment.

Conclusion: The results suggest that microbubbles can markedly improve the anti-cancer effect of DVDMS mediate sonodynamic therapy both in vitro and in vivo.
\end{abstract}

Key words: Microbubble, sinoporphyrin sodium, sonodynamic therapy, tumor.

\section{Introduction}

Sonodynamic therapy (SDT) is a vital alternative treatment modalities used for tumors in recent decades. SDT involves the synergistic effect of sonosensitizer and ultrasound on tumor damage [1]. Ultrasound, a kind of mechanical wave, can easily propagate through several centimeters of tissue with weak decay, and this property broadens SDT therapeutic range to deep seated malignant tumors [2]. The preferential accumulation of sonosensitizer in tumors and the tumor local ultrasound exposure contribute to targeted elimination of tumors [3]. In recent years, an ever-increasing amount of data verified the curative effect of SDT on treating tumors both in vitro and in vivo [4-6]. Moreover, in 2008 Wang et al. [7] reported three cases of metastasized pathologically breast cancer treated with SDT, and the primary clinical data showed that SDT is well tolerated and has a significant therapeutic effect for advanced breast cancer. In 2014, Toshio et al. [8] reported a case of terminal breast cancer patient treated with SDT combined with endocrinotherapy and immunotherapy, the significant therapeutic effect and good prognosis suggested 
the potential anti-cancer effect of combined therapies.

Sonosensitizer, a key component in the process of SDT, greatly affect SDT efficiency [9]. Many photosensitizers that used in photodynamic therapy are proved also can be activated by ultrasound eg, photofrin, haematoporphyrin, protoporphyrin, chlorin e6 and hypocrellin [10-14]. Sinoporphyrin sodium also called DVDMS, a novel photosensitizer, is depurate from photofrin II by Fang at Chinese Academy of Medical Sciences. DVDMS possesses excellent photoand sono-activities phototoxicity with tiny dark toxicity [14-17]. Further, the high chemical purity, good water solubility, explicit active ingredients [18], well tumor selectively accumulate and sono-/phototoxicity indicated the potential clinical application of DVDMS.

Microbubbles (MBs) are small gas-filled microspheres that widely used in medical diagnosis as contrast agents based on the specific acoustic properties [19]. Under low and intermediate acoustic pressure, MBs were forced expansion and compression and ultimate destruction, the shock waves generated in this process improve cellular membrane permeability by transiently perforating the plasma membranes [20]. Based on this mechanism, in recent years, MBs had been extensively used in drug or gene delivery and enhancing chemotherapy effect [21, 22]. SonoVue® is a suspension of stabilized sulfur hexafluoride (SF6) microbubbles which is isotonic to human plasma and very stable and resistant to pressure [23]. Many pre-clinical studies of animal models have shown the security and effectiveness of SonoVue®, and it has been widely used in clinic as contrast agent to enhance ultrasound imaging.

With the development of SDT, some adjuvant agents were introduced to enhance its curative effect. Nano-/lipidosome sonosensitizers can significantly improve the cytotoxicity and tumor inhibition rate induced by SDT [24-26]. In addition, chemotherapeutics and photodynamic therapy (PDT) also can enhance SDT effectiveness [27-31]. MBs used to improve drug delivery and chemotherapy synergism, however, scarce research on combination of SDT and MB are performed. Nomikou et al. [32] reported the enhanced cytotoxicity in cancer cell line and the greater tumor growth inhibition when the sonosensiter covalently link to a lipid microbubble, similarly, Ruan et al. [33] found that the combination of SDT and MB can significantly enhance the cytotoxic effect on MDA-MB-231 cells, their results indicated that the combination may be another modality to improve SDT effect.

In this study, we mainly focused on the effect of the co-administration of a clinically-approved MB and a novel sonosensitizer DVDMS mediate SDT on mice colorectal cancer both in vitro and in vivo.

\section{Materials and methods}

\section{Cell culture and animal model}

CT26 cells were obtained from Cell Resource Center, Chineses Academy of Medical Sciences, Beijing, China. Cells were cultured in RPMI 1640 medium (Sigma-Aldrich) supplemented with $10 \%$ fetal bovine serum (FBS, Hyclone, USA), $100 \mathrm{U} / \mathrm{ml}$ penicillin, $100 \mu \mathrm{g} / \mathrm{ml}$ streptomycin, and $1 \mathrm{mM}$ L-glutamine. Cultures were maintained at $37^{\circ} \mathrm{C}$ with humidity and $5 \% \mathrm{CO}_{2}$.

BALB/c mice (female, 18-20 g body weight) were supplied by the Experimental Animal Center of the Fourth Military Medical University (FMMU) (Xi'an, China). In the experiment, $1 \times 10^{6} \mathrm{CT} 26$ cells in $0.1 \mathrm{ml}$ physiological saline were subcutaneously injected into the left oxter region of BALB/c mice. When the tumors grew to about $5 \mathrm{~mm}$ in diameter, mice were ready for experiments. All experiments using live animals were carried out with approval from the university's Institutional Animal Care and Use Committee.

\section{Chemicals}

DVDMS was dissolved in phosphate buffer solution (PBS, pH 7.2-7.4), sterilized, aliquoted and stored in the dark at $-20^{\circ}$ C. The SonoVue ${ }^{\circledR}$ (Bracco, Milan, Italy) suspension was prepared in-situ using 5 $\mathrm{ml}$ of sterile saline ( $0.9 \%$ sodium chloride) according to the manufacturer's instructions and diluted at a ratio of 1:5 just before administration. 3-(4, 5-dimethylthiazol-2-yl)-2, 5-diphenyl tetrazolium bromide (MTT), fluorescein isothiocyanate-dextran (FD500). Guava Viacount Reagent and Guava Nexin Reagent were obtained from Guava Technologies (Hayward, CA).

\section{Ultrasound apparatus}

Ultrasonic setup for in vitro experiments is the same as previous [34]. The continuous ultrasound wave generated by a multi-functional generator (T\&C Power Conversion, Inc., USA) was used to treat cells. The continuous wave was outputted by a 35-mm-diameter planar transducer (Institution of Applied Acoustics, Shaanxi Normal University), which was submerged in an acrylic tank filled with distilled degassed water. To minimize reflected ultrasound, the end of the tank was covered with 6-mm-thick polyester films. For sonication, cells were exposed at intensities of $0.36 \mathrm{~W} / \mathrm{cm}^{2}, 0.54 \mathrm{~W} / \mathrm{cm}^{2}$ (ISATA) with the frequency of $1.0 \mathrm{MHz}$ for $0.5-1.0 \mathrm{mi}-$ nute.

It should be noted that for in vivo experiment a 
focused ultrasound transducer with a circular ceramic plate of $22 \mathrm{~mm}$ in diameter was used to treat tumor-bearing mice. The transducer was also submerged in cold degassed water. Under anesthesia with pentobarbital, a mouse with a left oxter-transplanted tumor was placed downward directly at the focal point for sonication with $\mathrm{LP}=4 \mathrm{~W}$ for 3 minutes with $1.9 \mathrm{MHz}$.

\section{Treatment protocols}

$5 \times 10^{5}$ CT26 cells were seeded into a $35-\mathrm{mm}$ culture dish and divided randomly into control, ultrasound alone, ultrasound plus MB, sensitizer alone, SDT and SDT plus MB groups. Cells were incubated with $1 \mu \mathrm{g} / \mathrm{ml}$ DVDMS for $4 \mathrm{~h}$ and then exposed to ultrasound. The diluted solution of SonoVue ${ }^{\circledR}$ (the final ratio of cell and SonoVue ${ }^{\circledR}$ number was 1:5) were added into dish just before ultrasound irradiation.

The tumor-bearing mice were randomly divided into six groups (6 mice in each group) which was the same as cell experiments. $2 \mathrm{mg} / \mathrm{kg}$ DVDMS was injected into the mice through caudal vein, $2 \mathrm{~h}$ after DVDMS infusion, $100 \mu \mathrm{L} / \mathrm{kg}$ SonoVue ${ }^{\circledR}$ was injected through caudal vein. After SonoVue ${ }^{\circledR}$ injection, mice were exposed to the focused ultrasound spot for 3 minutes at $4 \mathrm{~W}$ load power with $1.90 \mathrm{MHz}$ frequency. All experiments were performed in the dark to avoid DVDMS excitation.

\section{Cytotoxicity analysis}

The cytotoxicity was analyzed using MTT and Guava Viacount assay, as previously described [35]. Cell survival was calculated using the following equation:

$$
\begin{aligned}
\text { Cell survival }(\%)= & \text { OD treatment group/OD control } \\
& \text { group } \times 100 \%
\end{aligned}
$$

\section{Apoptosis detection}

Annexin V-FICT and PI detection Kit (Invitrogen, USA) was used to quantify cell apoptosis. Apoptotic cells were measured according to the manufacturer's protocol. Briefly, after different treatment, cells were harvested by trypsinization, cell pellets were suspended in $1 \times$ binding buffer and stained with $5 \mu \mathrm{l}$ of annexin V-FITC and $5 \mu \mathrm{l}$ of PI for 10 minutes. After staining, samples were investigated using flow cytometry.

\section{Cell membrane permeability study}

FD500 is a kind of macromolecule that combine fluoresce in isothiocyanate (FITC) with dextran. The high molecular weight $(500,000)$ of FD500 make it difficult to enter into cell membrane only when cell membrane permeability increased. Cells were treated with ultrasound in the presence of $1 \mathrm{mg} / \mathrm{ml}$ of FD500. After ultrasound treatment, cells were washed with PBS twice, then immediately analyzed by flow cytometry. FD500 fluorescent-positive cells were calculated by flow cytometry and data was analyzed using FCS Express software.

\section{Increased DVDMS uptake detection}

Flow cytometry was applied to quantify the intracellular DVDMS content. After different treatment, cells were harvested by trypsinization and immediately washed with PBS, subsequently the intracellular DVDMS fluorescence intensity measured by flow cytometry.

\section{Evaluation of anti-tumor effect}

Nine days after treatment, mice in different groups sacrificed and tumors were stripped. The therapeutic results were measured by the tumor weight. The weight inhibition ratios were calculated as: \{1-(average tumor weight of treated group/average tumor weight of the control group) $\} \times$ $100 \%$. Moreover, a representative mice and the corresponding tumor in each groups were captured.

\section{Histological examination by H\&E staining}

Tumor tissues in different groups were fixed with $10 \%$ buffered formalin for $24 \mathrm{~h}$. Subsequently, samples were paraffin-embedded, sectioned, and stained with hematoxylin and eosin (H\&E). Histopathological changes were observed under microscope (Nikon E600).

\section{Statistical analysis}

All values are expressed as the mean \pm standard deviation. Differences between the treatment groups were assessed with one-way analysis of variance. Statistical significance was established at a P-value < 0.05 . Each experiment was repeated three times.

\section{Results}

\section{Cytotoxicity analysis}

As shown in Figure 1A, DVDMS inhibited CT26 cell proliferation in a dose- and incubation time-dependent manner. At $4 \mathrm{~h}$ after treatment, the cell survival rate was $95.65 \%(p>0.05), 97.40 \%$ $(p>0.05), 96.31 \%(p>0.05), 94.60 \%(p>0.05)$ and 81.74 $\%(\mathrm{p}>0.05)$, as the incubation time prolong to $24 \mathrm{~h}$ the survival rate decreased to $95.47 \%(p>0.05), 84.68 \%$ $(p>0.05), 80.13 \%(p<0.05), 67.96 \%(p>0.01)$ and 63.35 $\%(\mathrm{p}>0.01)$ with the DVDMS concentration was $0.5,1$, 2,4 and $8 \mu \mathrm{g} / \mathrm{ml}$, respectively. Figure $1 \mathrm{~B}$ showed the cell viability after ultrasound irradiation. $0.36 \mathrm{~W} / \mathrm{cm}^{2}$ ultrasound didn't cause significant cell proliferation inhibition. The cell viability decreased to $87.77 \%$ 
( $>>0.05), 84.81 \%(p>0.05)$ (4 h after treatment) and $86.98 \%(p>0.05), 79.01 \%(p<0.05) \quad(24 \mathrm{~h}$ after treatment) when the ultrasound intensity was $0.54 \mathrm{~W} / \mathrm{cm}^{2}$ with 0.5 and 1 minute exposure time, respectively. Moreover, the cell viability loss caused by SDT was showed in Figure 1C, the combination of $1 \mu \mathrm{g} / \mathrm{ml}$ DVDMS and $0.54 \mathrm{~W} / \mathrm{cm}^{2}$ ultrasound caused $24.70 \%$ $(\mathrm{p}<0.01), 60.59 \%(\mathrm{p}<0.01)(4 \mathrm{~h}$ after treatment $)$ and $41.54 \%(\mathrm{p}<0.01), 73.25 \%(\mathrm{p}<0.01)(24 \mathrm{~h}$ after treatment) viability decrease when the ultrasound irradiation time was 0.5 and 1 minute, respectively.

With the introduction of MBs to ultrasound and SDT treatment, additional cytotoxicity was found (Figure 1D). MBs alone didn't show any cytotoxicity, but in the presence of MBs, cell growth inhibition rate caused by ultrasound increased from $9.52 \%$ to 25.35 $\%(\mathrm{p}<0.05)(4 \mathrm{~h}$ after treatment) and $7.45 \%$ to $23.21 \%$ $(\mathrm{p}<0.05)$ (24 h after treatment). For SDT plus MBs groups, the cell viability decreased to $49.47 \%(\mathrm{p}<0.01)$ ( $4 \mathrm{~h}$ after treatment) and $38.72 \%(\mathrm{p}<0.01)$ ( $24 \mathrm{~h}$ after treatment).

\section{Viacount assay}

Guava Viacount assay was applied to confirm the cell viability in different treatment groups, and the results were shown in Figure 2. According to instruc- tions, living cells were on the left side of the plot, while damaged cells were on the right. Compared with control group (97.37\%), cell viability in $1 \mu \mathrm{g} / \mathrm{ml}$ DVDMS, $0.54 \mathrm{~W} / \mathrm{cm}^{2}$ with 0.5 minute ultrasound and SDT groups decreased to $93.80 \%, 90.36 \%$ and 81.39 $\%$, respectively, while the addition of MBs in ultrasound and SDT group caused an additional cytotoxicity with survival rate of $86.14 \%$ and $62.67 \%$, respectively. The results further confirmed the synergistic effect of MBs and ultrasound and SDT. The experiment treatment parameters were used in the next test.

\section{Apoptosis detection}

The apoptosis rate was studied with Annexin V-FITC and PI staining after various treatments. Cells in the lower-left quadrant represented viable cells, both the lower-right and upper-right quadrant showed the apoptotic cells, and the necrotic cells appeared in the upper-left quadrant. As shown in Figure 3 , there are $95.55 \%, 91.55 \%$ and $94.30 \%$ viable cells in control, DVDMS and ultrasound groups, respectively. SDT can mainly induce cell apoptosis with the apoptosis ratio was $16.53 \%$. In addition, $5.8 \%$ apoptotic and $9.10 \%$ necrotic cells were detected in ultrasound plus MBs group. MBs plus SDT treatment caused $12.60 \%$ apoptosis and $26.95 \%$ necrotic cells.
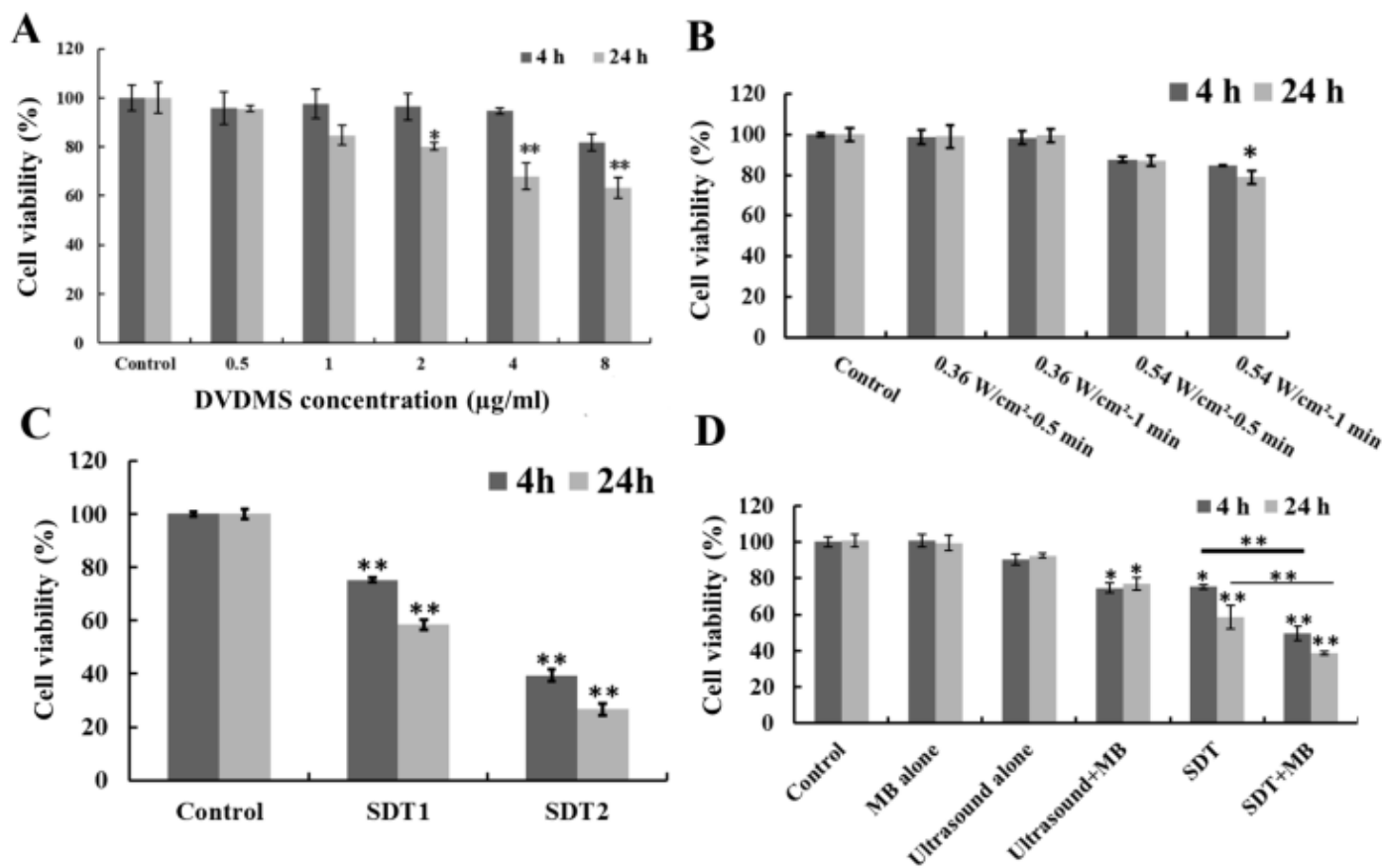

Figure 1. Viability of CT26 cells after different treatment. Different DVDMS concentration (A) and ultrasound parameters (B); SDT treatment (C), SDT1: $1 \mu \mathrm{g} / \mathrm{ml}$ combined with $0.54 \mathrm{~W} / \mathrm{cm}^{2}$ with 0.5 minute ultrasound, SDT2: $1 \mu \mathrm{g} / \mathrm{ml}$ combined with $0.54 \mathrm{~W} / \mathrm{cm}^{2}$ with 1 minute ultrasound; SDT combined with MBs (D) cytotoxicity. Ultrasound: $0.54 \mathrm{~W} / \mathrm{cm}^{2}$ with 0.5 minute; SDT: $1 \mu \mathrm{g} / \mathrm{ml}$ combined with $0.54 \mathrm{~W} / \mathrm{cm}^{2}$ with 0.5 minute ultrasound; The ratio of MBs and cells was $5: 1$ in Ultrasound+MBs and SDT+MBs groups. Cell viability was measured by MTT assay. All data are expressed as percentage of untreated cells, error bars represent S.D. of the means from three independent experiments. ${ }^{*} \mathrm{p}<0.05$ and $* * \mathrm{p}<0.01$ versus untreated cells. 

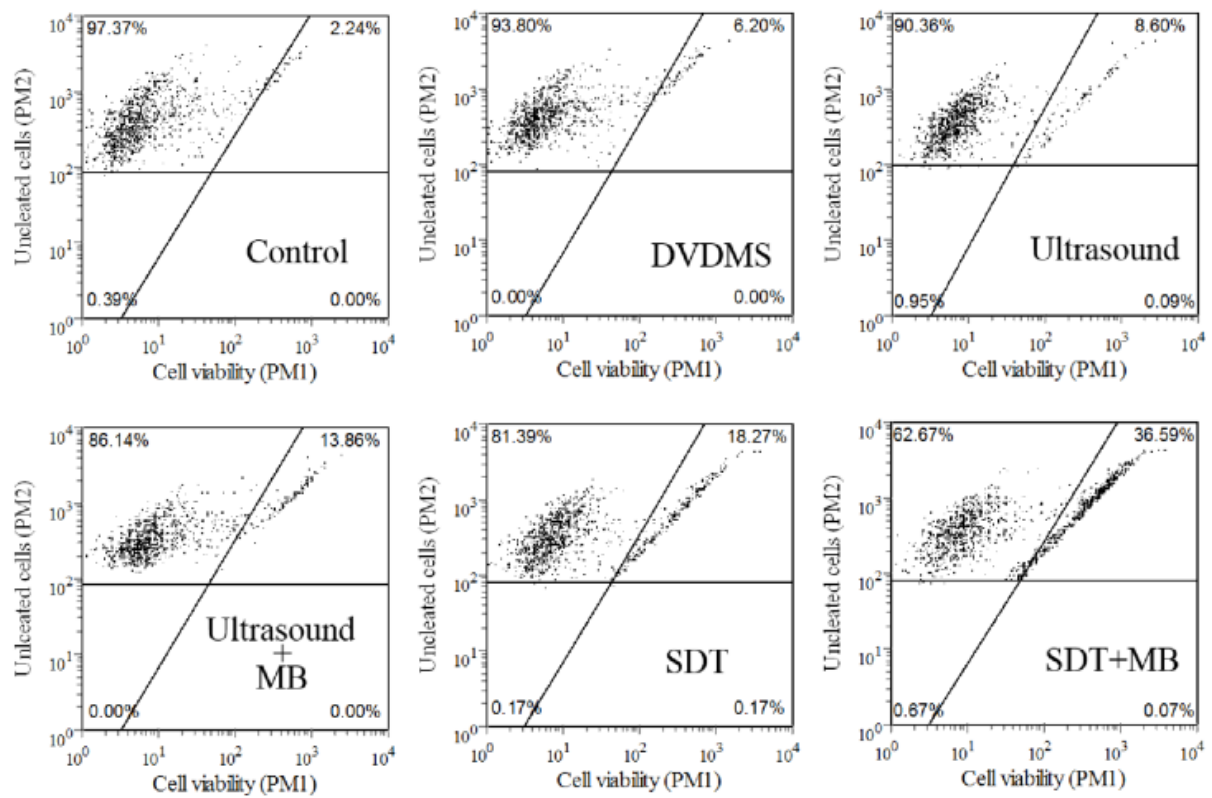

Figure 2. Analysis of CT26 cells viability by Guava Viacount assay. DVDMS: $1 \mu \mathrm{g} / \mathrm{ml}$ Ultrasound: $0.54 \mathrm{~W} / \mathrm{cm}^{2}$ with $0.5 \mathrm{minute}$; SDT: $1 \mu \mathrm{gg} / \mathrm{ml}$ combined with $0.54 \mathrm{~W} / \mathrm{cm}^{2}$ with 0.5 minute ultrasound; The ratio of MBs and cells equaled 5:1 in Ultrasound+MBs and SDT+MBs groups.
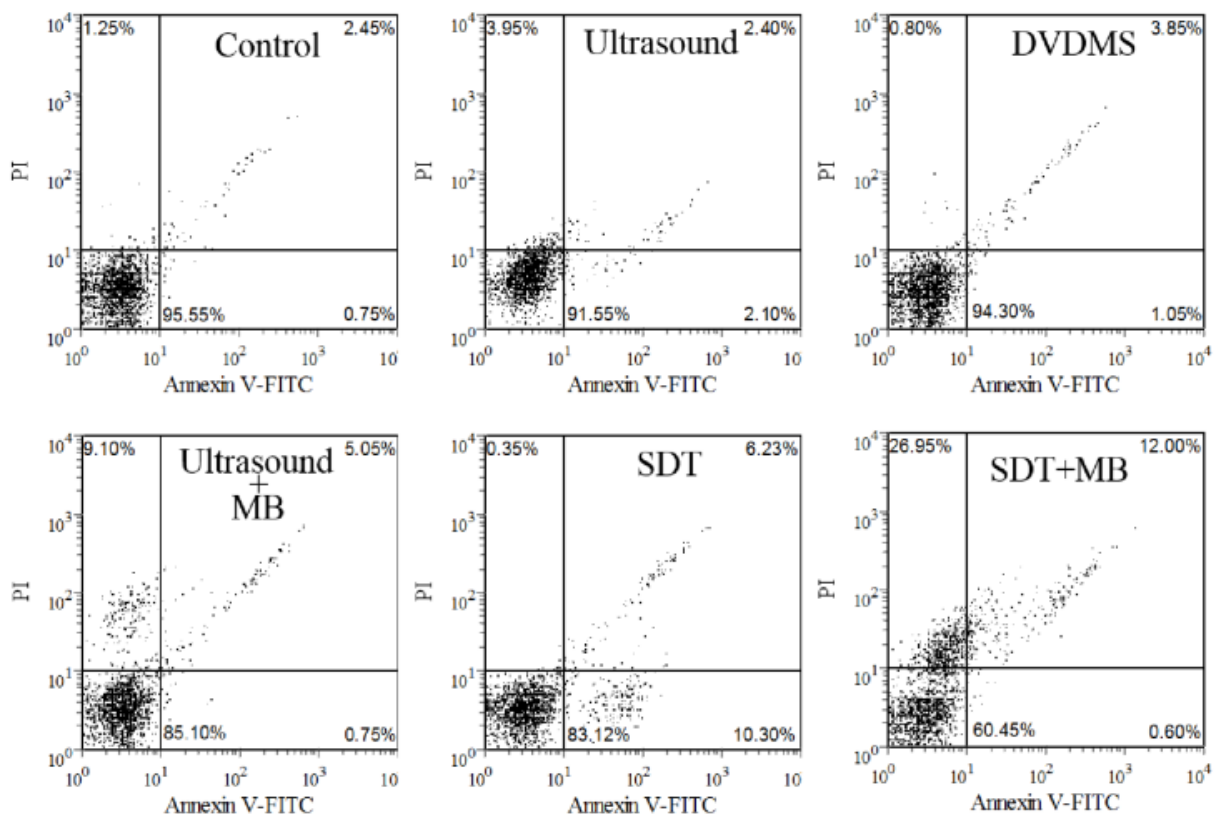

Figure 3. Dot plots of Annexin-FITC and PI uptake by different treated cells.

\section{Increased cell membrane permeability study}

The change of cell membrane permeability after ultrasound treatment with/without the MBs was measured by FD500. Intracellular fluorescence intensity of FD500 indirectly reflected the level of membrane permeability. As illustrated in Figure 4, compare with control groups green fluorescence of FD500 has a significant increase in SDT group, while with the addition of MBs the intracellular green fluorescence intensity was further enhancement.

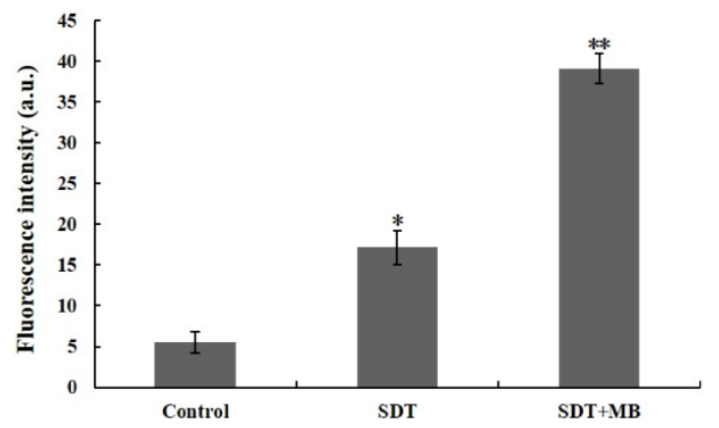

Figure 4. Detection of CT26 cell membrane permeability. Immediately after ultrasound exposure in the presence of macromolecule FD500, the FD500 fluorescent positive cells were evaluated by flow cytometry. Data are means \pm SD of three independent experiments. ${ }^{*} p<0.05$ and ${ }^{*}{ }^{*} p<0.01$, compared with control group. 
A

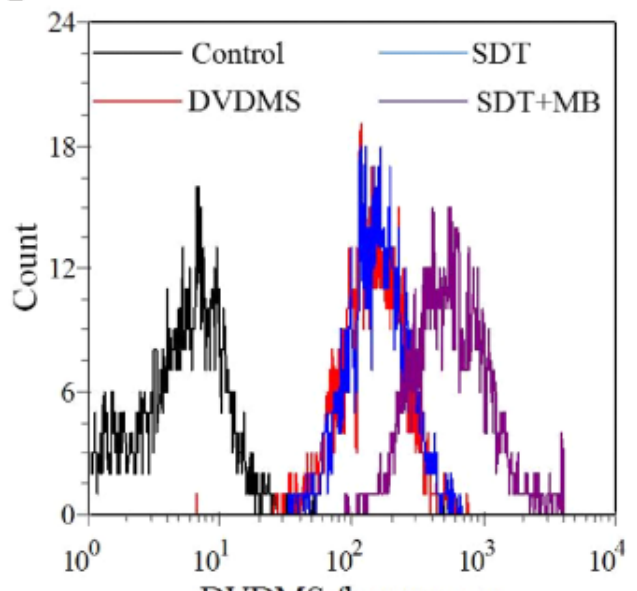

$\mathrm{B}$

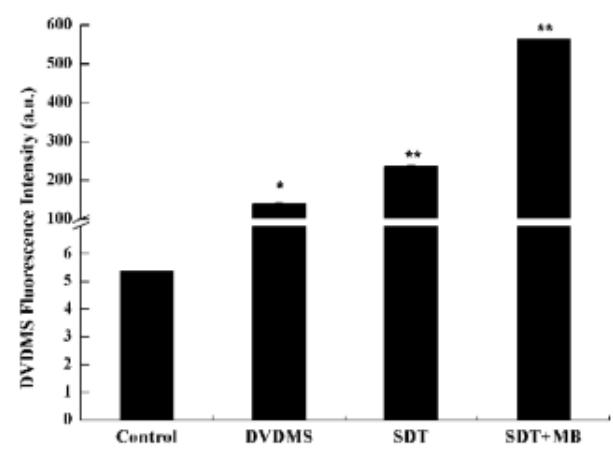

DVDMS fluorescence

Figure 5. DVDMS accumulation in CT26 cells. Immediately following different treatment, the DVDMS fluorescent positive cells were measured by flow cytometry. Measurement of fluorescence intensity by flow cytometry $(A)$ and the recorded mean fluorescence intensities (B). Data are presented as mean \pm SD from three independent experiments. ${ }^{*} p<0.05$ and ${ }^{* *} p<0.01$ compare with DVDMS group.

Increased DVDMS uptake detection after different treatment

The uptake of DVDMS in CT26 cells was analyzed by flow cytometry, and the corresponding results were illustrated in Figure 5. Compare with the DVDMS control, cells treated with SDT can uptake more DVDMS, and a remarkable improvement of DVDMS uptake was found in SDT plus MBs group (Figure 5a). Figure $5 \mathrm{~b}$ demonstrated the specific DVDMS fluorescence intensity, intracellular DVDMS content was 1.7 and 4.0 fold higher in SDT and SDT plus MB groups than DVDMS control group, respectively.

\section{Evaluation of the anti-tumor effect}

After treatment, the tumor weight at $9^{\text {th }}$ day after treatment was demonstrated at Figure 6 and Table 1. Visualized inhibition effect was displayed in Figure 6. The tumor weight inhibition ratios were $11.17 \%$ $(\mathrm{p}>0.05)$ and $21.87 \%(\mathrm{p}<0.05)$ in DVDMS $(2 \mathrm{mg} / \mathrm{kg})$ and ultrasound groups $(\mathrm{LP}=4 \mathrm{~W})$, respectively, and the inhibition ratio increased to $44.38 \%(\mathrm{p}<0.01)$ in SDT treatment group. While, with the introduction of MBs the tumor inhibition effect significantly increased to $42.68 \%(\mathrm{p}<0.01)$ and $60.00 \%(\mathrm{p}<0.01)$ in ultrasound + MBs, and SDT + MBs groups, respectively.

The weight curve of mice in Figure 7 indirectly reflected the therapeutic effect of different treatments. In the whole therapeutic process, the mice weight have no remarkable change both in control and different treatment groups, indicating pimping side effects of the treatments.

\section{Visual observation}

At $9^{\text {th }}$ day after treatment, a tumor-bearing mouse in each group was photographed and the cor- responding tumor was showed in Figure 8. The tumor volume in ultrasound, ultrasound + MB, SDT, SDT + $\mathrm{MB}$ groups were much smaller than control groups which was be consistent with tumor inhibition rate data in Table 1.

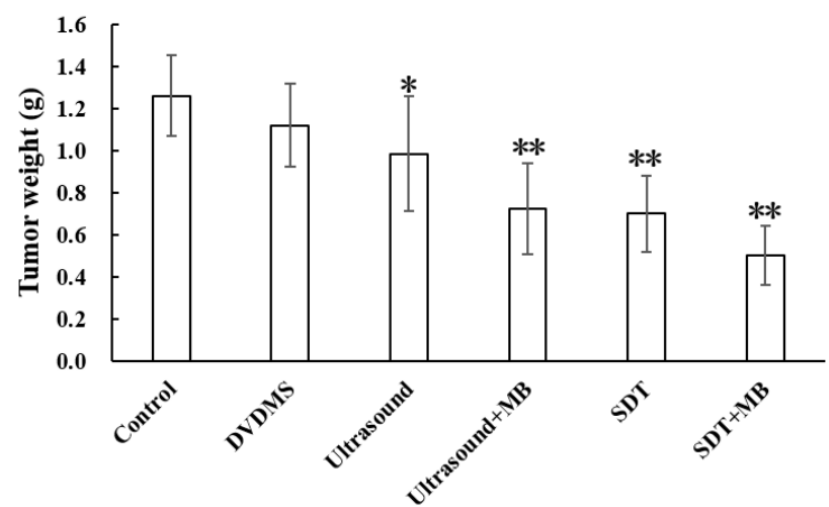

Figure 6. Tumor weight of CT26 tumor-bearing mice 9 days after different treatment. Control: untreated tumors; DVDMS: $2 \mathrm{mg} / \mathrm{kg}$ DVDMS alone; Ultrasound: tumor exposed to ultrasound ( $\mathrm{LP}=4 \mathrm{~W}, 3$ minutes); Ultrasound + MBs: $4 \mathrm{~W}$ with 3 minutesultrasound plus MBs; SDT: tumor treated with ultrasound in the presence of $2 \mathrm{mg} / \mathrm{kg}$ DVDMS; SDT + MBs: tumor treated with ultrasound in the presence of 2 $\mathrm{mg} / \mathrm{kg}$ DVDMS and MBs.

Table 1. Tumor weight and inhibition rate of different treatment on CT26 tumors

\begin{tabular}{cccc}
\hline Groups & $\mathbf{n}$ & Weight of tumor $(\mathbf{g})$ & Inhibition rate (\%) \\
\hline Control & 6 & $1.264 \pm 0.195$ & 0.000 \\
DVDMS & 6 & $1.122 \pm 0.198$ & 11.166 \\
Ultrasound & 6 & $0.987 \pm 0.274$ & $21.869 *$ \\
Ultrasound + MB & 6 & $0.723 \pm 0.216$ & $42.681^{* *}$ \\
SDT & 6 & $0.701 \pm 0.183$ & $44.382 * *$ \\
SDT + MB & 6 & $0.504 \pm 0.140$ & $60.000^{* *}$ \\
\hline
\end{tabular}




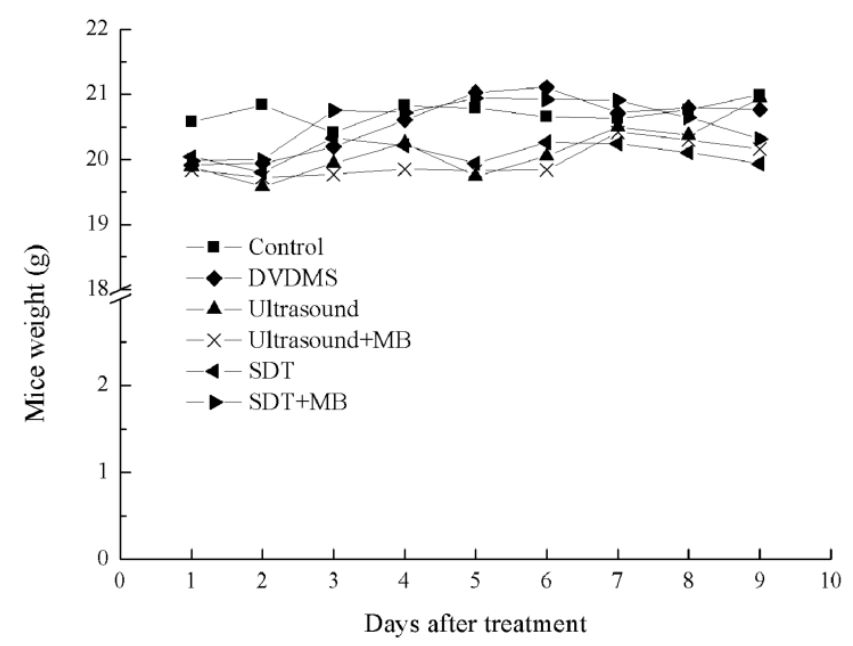

\section{Histological observation}

H\&E staining was used to observe the tumor tissues after different treatments (Figure 9). Tissue in the untreated group showed compact tumor cells with intact structure and plump cell nucleus. Tumor cells in DVDMS and ultrasound groups were partly sparse and separated from each other. Obvious vacant sections and lots of nuclear fragments were founded in ultrasound + MBs and SDT treatment groups, further, the structure of tumor tissue were more seriously damaged in SDT + MBs group, most cells disintegrated and more cell fragments and vacant sections appeared.

Figure 7. The weight curve of CT26 tumor-bearing mice.

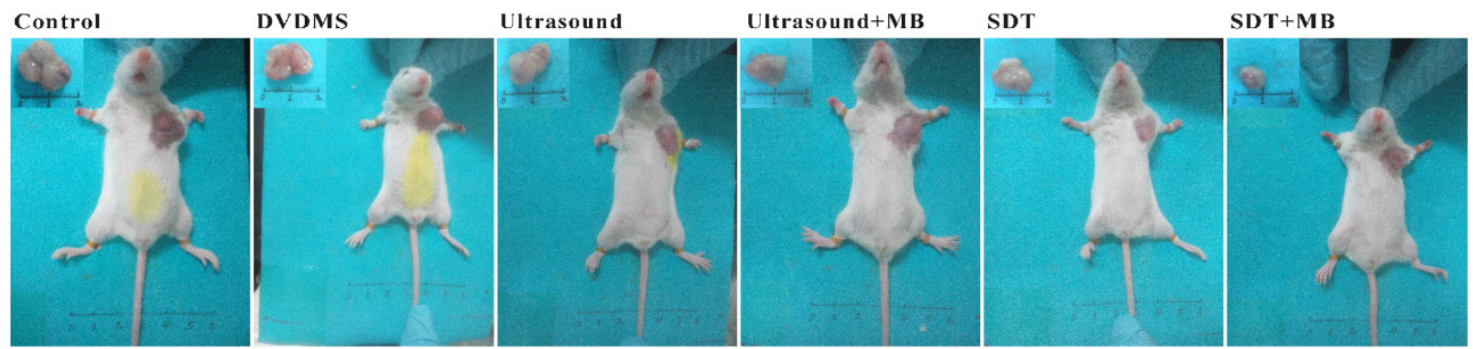

Figure 8. Pictures of mice and corresponding tumors in different groups 9 th after treatment.
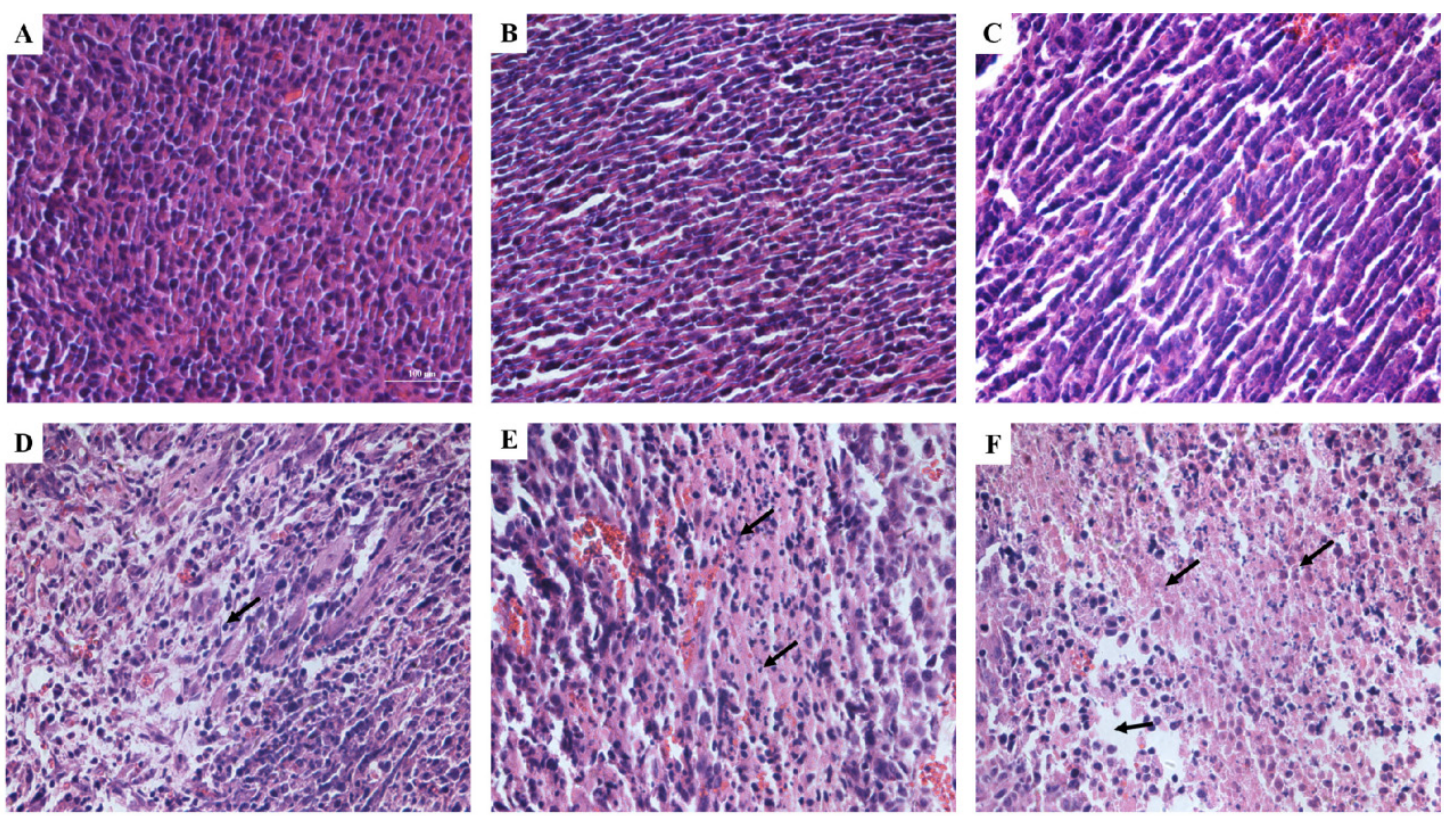

Figure 9. Tumor tissue structural changes of CT26 in mice. A: Control; B: DVDMS; C: ultrasound alone; D: ultrasound + MBs; E: SDT; F: SDT + MBs. See caption to Figure 7 for definitions of Control, DVDMS, ultrasound alone, ultrasound + MBs, SDT and SDT + MBs.

\section{Discussion}

Since the first report of SDT, a substantial amount of researchers began to investigate the anticancer effect and mechanism of SDT. For the past few years, some successful clinical cases of SDT treated terminally ill patient promoted the application of SDT [7]. Besides, the combination of SDT and other therapies such as chemotherapy or photodynamic therapy also manifest good anti-cancer effect [27-31], espe- 
cially for the combination of SDT and PDT (SPDT). In 2009, Kenyon reported 115 SPDT cases with remarkable therapeutic effect and good tolerance for various cancers [36]. MBs, usually applied as contrast agents to improve ultrasound imaging [37], nowadays also widely used in the drug delivery and targeted release system [38] by augmenting plasma membrane permeability [39]. Combined MBs with low energy ultrasound could kill cancer cells effectively and induce cell apoptosis [40]. However, studies about the combination of SDT and MBs are very rare. In this study, we mainly discussed the combination effect of DVDMS mediated SDT and clinically-approved MB, SonoVue ${ }^{\circledR}$, results in this paper could add to our knowledge and provide some ideas for the clinical application of this new approach for treating cancers.

Sensitizers as a kind of anticancer drugs may showed cytotoxicity without activation by light or ultrasound [41]. The toxicity of DVDMS on CT26 cells exerts concentration dependent manner according to Figure 1A. Moreover, ultrasound inhibit cell proliferation in an ultrasound intensity and treatment time-dependent manner. Based on these results, 1 $\mu \mathrm{g} / \mathrm{ml}$ DVDMS and $0.54 \mathrm{~W} / \mathrm{cm}^{2}$ with 0.5 minute ultrasound exposure, showing no significant cytotoxicity, were used in SDT treatment. The remarkable synergistic effect of DVDMS and ultrasound was demonstrated in Figure 1C. With the addition of MBs to the experiment system, further cell viability decrease was detected as illustrated in Figure 1D. The result of Viacount assay (Figure 2) confirmed the effectiveness of MBs.

Ultrasound-stimulated microbubbles caused rapid and extensive cytotoxicity on CT26 cells. Obvious cell apoptosis was observe in both ultrasound and SDT treatment groups, and with the introduction of MBs, cell necrosis rate and debris increased significantly, which was consistent with other literature [42]. The results indicated that MBs may enhanced the mechanically damage of ultrasound to tumor cells and excessive mechanical stimulation induce cell necrosis.

MBs, including SonoVue ${ }^{\circledR}$ that used in our current practice, were verified applied in improving drug delivery and chemotherapy efficiency by enhancing transient membrane permeabilization and intracellular drug accumulation [21, 22]. In order to analyze the influence on membrane permeabilization by ultrasound and MBs, intracellular green fluorescence of FD500 was detected. The enhancement of green fluorescence in ultrasound and SDT groups with the presence of MBs manifested the increasing of cell membrane permeabilization (Figure 4), the transient enhancement of permeabilization facilitated the absorbance of DVDMS in CT26 cells (Figure 5) which may contribute to the enhancement of cytotoxicity in SDT + MBs group.

Besides the analysis of therapeutic effect in vitro, the tumor inhibition rate was also measured in CT26 tumor-bearing mice. Results in Table 1 clearly illuminated the superiority of tumor inhibit effect in ultrasound + MBs and SDT + MBs treatment groups compare with the corresponding groups without MBs. One of the important advantages of SDT was the lower side effects compared with traditional cancer therapies [43, 44], and in the present study mice weight was statistical (Figure 7), there were no remarkable changes of mice weight in different treatment groups compare with control, suggesting slight side effects of this anti-cancer method. H\&E staining (Figure 9) was applied to demonstrate the damage level of different treatments. Obvious tissue destruction with cell debris and large spaces were observed after ultrasound plus MBs and SDT treatments. Moreover, the combination of SDT and MBs can further enhance the degree injury.

In conclusion, this study demonstrated the synergistic efficacy of SonoVue ${ }^{\circledR}$ and DVDMS-SDT both in vitro and in vivo. MBs improved necrocytosis via mechanically perturb cell membranes resulting in more accumulation of sensitizer in cells. Ultimately, the combination therapy showed significantly anti-cancer effect.

\section{Acknowledgments}

This research was supported by the National Natural Science Foundation of China (No. 81472846), the Fundamental Research Funds for the Central Universities (GK201502009, GK201504010) and the Excellent Doctor Innovation Project of Shaanxi Normal University (S2014YB03).

\section{Competing Interests}

The authors have declared that no competing interest exists.

\section{References}

1. Yumita N, Umemura S. Sonodynamic antitumour effect of chloroaluminum phthalocyanine tetrasulfonate on murine solid tumour. J phar pharmacol. 2004; 56: 85-90.

2. Milowska K, Gabryelak T. Synergistic effect of ultrasound and phthalocyanines on nucleated erythrocytes in vitro. Ultrasound in Med. \& Biol. 2005; 31: 1707-12.

3. Yumita N, Okudaira K, Momose Y, et al. Sonodynamically induced apoptosis and active oxygen generation by gallium-porphyrin complex, ATX-70. Cancer Chemother Pharmacol. 2010; 66: 1071-8.

4. Li Y, Su X, Wang X, et al. Cytotoxic effect of protoporphyrin IX to human Leukemia U937 cells under ultrasonic irradiation. Cell Physiol Biochem. 2014; 33: 1186-96.

5. Zheng L, Sun X, Zhu X, et al. Apoptosis of THP-1 derived macrophages induced by sonodynamic therapy using a new sonosensitizer hydroxyl acetylated curcumin. PLoS One. 2014; 9: e93133.

6. Tsuru H, Shibaguchi $\mathrm{H}$, Kuroki M, et al. Tumor growth inhibition by sonodynamic therapy using a novel sonosensitizer. Free Radic Biol Med. 2012; 53: 464-72. 
7. Wang X, Mitchell D, Lewis T. Primary clinical use of sonodynamic therapy (SDT) for advanced breast cancer. Journal of Clinical Oncology. 2008; 26: 12029.

8. Toshio I, Kaori M, Hirona M, et al. Case Report: A Breast Cancer Patient Treated with GcMAF, Sonodynamic Therapy and Hormone Therapy. Anticancer Research. 2014; 34: 4589-94.

9. Bellnier D, Ho Y, Pandey R, et al. Distribution and elimination of Photofrin II in mice. Photochem Photobiol. 1989; 50: 221-8.

10. Xu Z, Wang K, Li X, et al. The ABCG2 transporter is a key molecular determinant of the efficacy of sonodynamic therapy with Photofrin in glioma stem-like cells. Ultrasonics. 2013; 53: 232-8.

11. Wang H, Liu Q, Zhang $\mathrm{K}$, et al. Comparison between sonodynamic and photodynamic effect on MDA-MB-231 cells. J Photochem Photobiol B. 2013; 5: 182-191.

12. Liu $Q$, Wang $X$, Wang $P$, et al. Comparison between sonodynamic effect with protoporphyrin IX and hematoporphyrin on sarcoma 180. Cancer Chemother Pharmacol. 2007; 60: 671-80.

13. Wang $\mathrm{X}$, Leung $\mathrm{AW}$, Jiang $\mathrm{Y}$, et al. Hypocrellin B-mediated sonodynamic action induces apoptosis of hepatocellular carcinoma cells. Ultrasonics. 2012; 52: $543-6$

14. Guo S, Sun X, Cheng J, et al. Apoptosis of THP-1 macrophages induced by protoporphyrin IX-mediated sonodynamic therapy. Int J Nanomedicine. 2013; 8: 2239-46.

15. Jiang Z, Shi R, Li C, et al. Inhibitory effects of DVDMS-2-based-photodynamic therapy on the growth of tumor and in vitro in vivo. Teratog Carcinog Mutagen. 2013; 25: 163-7.

16. Li C, Zhang K, Wang P, et al. Sonodynamic antitumor effect of a novel sonosensitizer on S180 solid tumor. Biopharm Drug Dispos. 2014; 35: 50-9.

17. Hu J, Wang $X$, Zhang $K$, et al. Sinoporphyrin sodium: a novel sensitizer in sonodynamic therapy. Anticancer Drugs. 2014; 25: 174-82

18. Fang Q, Yang D. A porphyrin dimer combined with an ether bond and its manufacturing method. Patent ZL200910179116.5. People's Republic of China. 2012.

19. Dijkmans P, Juffermans L, Musters $R$, et al. Microbubbles and ultrasound: from diagnosis to therapy. Eur J Echocardiogr. 2004; 5: 245-56.

20. Rapoport N, Kennedy A, Shea J, et al. Controlled and targeted tumor chemotherapy by ultrasound-activated nanoemulsions/microbubbles. J Control Release. 2009; 138: 268-76.

21. Yoon $\mathrm{Y}, \mathrm{K}$ won $\mathrm{Y}, \mathrm{Cho} \mathrm{H}$, et al. Ultrasound-mediated gene and drug delivery using a microbubble-liposome particle system. Theranostics. 2014; 4: 1133-44.

22. Yang $S$, Wang $P$, Wang $X$, et al. Activation of microbubbles by low-level therapeutic ultrasound enhances the antitumor effects of doxorubicin. Eur Radiol. 2014; 24: 2739-53.

23. Nicolau C, Catalá V, Vilana R, et al. Evaluation of hepatocellular carcinoma using SonoVue ${ }^{\circledR}$, a second generation ultrasound contrast agent: correlation with cellular differentiation. Eur Radiol. 2004; 14: 1092-9.

24. Shanei A, Sazgarnia A, Tayyebi, et al. Sonodynamic Therapy Using Protoporphyrin IX Conjugated to Gold Nanoparticles: An In Vivo Study on a Colon Tumor Model. Iran J Basic Med Sci. 2012; 15: 759-67.

25. Sazgarnia A, Shanei A, Meibodi N, et al. A novel nanosonosensitizer for sonodynamic therapy: in vivo study on a colon tumor model. J Ultrasound Med. 2011; 30: 1321-9.

26. Canaparo R, Varchi G, Ballestri M, et al. Polymeric nanoparticles enhance the sonodynamic activity of meso-tetrakis (4-sulfonatophenyl) porphyrin in an in vitro neuroblastoma model. Int J Nanomedicine. 2013; 8: 4247-63.

27. Liang L, Xie S, Jiang L, et al. The Combined effects of hematoporphyrin monomethyl ether-SDT and doxorubicin on the proliferation of QBC939 cell lines. Ultrasound Med Biol. 2013; 39: 146-60.

28. Li Q, Wang $X$, Wang $P$, et al. Efficacy of chlorin e6-mediated sono-photodynamic therapy on $4 \mathrm{~T} 1$ cells. Cancer Biother Radiopharm. 2014; 29: $42-52$.

29. Tserkovsky D, Alexandrova E, Chalau V, et al. Effects of combined sonodynamic and photodynamic therapies with photolon on a glioma C6 tumor model. Exp Oncol. 2012; 34: 332-5.

30. Wang $\mathrm{X}$, Zhang $\mathrm{W}, \mathrm{Xu} \mathrm{Z}$, et al. Sonodynamic and photodynamic therapy in advanced breast carcinoma: a report of 3 cases. Integr Cancer Ther. 2009; 8: 283-7.

31. Jin Z, Miyoshi N, Ishiguro K, et al. Combination effect of photodynamic and sonodynamic therapy on experimental skin squamous cell carcinoma in C3 H/HeN mice. J. Dermatol. 2000; 27: 294-306.

32. Nomikou N, Fowley C, Byrne NM, et al. Microbubble-sonosensitiser conjugates as therapeutics in sonodynamic therapy. Chem Commun (Camb). 2012; 48: $8332-4$

33. Ruan J, Wu H, Xu XL, et al. Synergy of hematoporphyrin monomethyl ether and SonoVue ${ }^{\circledR}$ on the killing of MDA-MB-231 cells under ultrasound. Chin J Med Imaging Technol. 2013; 29: 165-8.

34. Wang $\mathrm{H}$, Wang $\mathrm{X}$, Wang $\mathrm{P}$, et al. Ultrasound enhances the efficacy of chlorin E6-mediated photodynamic therapy in MDA-MB-231 cells. Ultrasound Med Biol. 2013; 39: 1713-24.

35. Yang S, Wang P, Wang X, et al. Efficacy of combined therapy with paclitaxel and low-level ultrasound in human chronic myelogenous leukemia cell line K562. J Drug Target. 2013; 21: 874-84.

36. Kenyon J, Fuller R, Lewis T. Activated cancer therapy using light and ultrasound-a case series of sonodynamic photodynamic therapy in 115 patients over a 4 years period. Current Drug Therapy. 2009; 4: 179-93.
37. Shen Y, Zhang A, Guo J, et al. Fluorescence imaging of Evans blue extravasation into mouse brain induced by low frequency ultrasound with microbubble. Biomed Mater Eng. 2014; 24: 2831-8.

38. Meijering B, Juffermans L, Van Wamel, et al. Ultrasound and microbubble-targeted delivery of macromolecules is regulated by induction of endocytosis and pore formation. Circ Res. 2009; 104: 679-87.

39. Zhao Y, Gao H, Zhou Z, et al. Experiment on the factors for enhancing the susceptibility of cancer cells to chemotherapeutic drug by ultrasound microbubbles. J Drug Target. 2010; 18: 430-7.

40. Zhang B, Zhou $\mathrm{H}$, Cheng $\mathrm{Q}$, et al. Low-frequency low energy ultrasound combined with microbubbles induces distinct apoptosis of A7r5 cells. Mol Med Rep. 2014; 10: 3282-8.

41. Bednarz N, Zawacka-Pankau J, Kowalska A. Protoporphyrin IX induces apoptosis in HeLa cells prior to photodynamic treatment. Pharmacol Rep. 2007; 59: 474-9.

42. Escoffre J, Piron J, Novell A, et al. Doxorubicin delivery into tumor cells with ultrasound and microbubbles. Mol Pharm. 2011; 8: 799-806.

43. Tachibana K, Feril LB Jr, Ikeda-Dantsuji Y. Sonodynamic therapy. Ultrasonics. 2008; 48: 253-9.

44. Shibaguchi H, Tsuru H, Kuroki M, et al. Sonodynamic Cancer Therapy: A Non-invasive and Repeatable Approach Using Low-intensity Ultrasound with Sonosensitizer. Anticancer Res. 2011; 31: 2425-9. 